\title{
COLGAJO DE PECTORAL MAYOR PARA RECONSTRUCCIÓN DE DEFECTOS FARÍNGEOS. DESCRIPCIÓN DE UN CASO
}

\section{Pectoralis major myocutaneous flap for faringeal defects reconstruction. Case report}

\author{
Cristina Isabel SANZ-SÁNCHEZ; Else KRAEMER-BAEZA; Eva FLORES-CARMONA; María \\ Dolores AGUILAR-CONDE; Óscar Emilio CAZORLA-RAMOS
}

Hospital Universitario Virgen de la Victoria. Málaga.

Correspondencia: cristinasanzsanchez03@gmail.com

Fecha de recepción: 23 de junio de 2020

Fecha de aceptación: 3 de septiembre de 2020

Fecha de publicación: 5 de septiembre de 2020

Fecha de publicación del fascículo: pendiente de publicación

Conflicto de intereses: Los autores declaran no tener conflictos de intereses Imágenes: Los autores declaran haber obtenido las imágenes con el permiso de los pacientes Política de derechos y autoarchivo: se permite el autoarchivo de la versión post-print (SHERPA/RoMEO) Licencia CC BY-NC-ND. Licencia Creative Commons Atribución-NoComercial-SinDerivar 4.0 Internacional Universidad de Salamanca. Su comercialización está sujeta al permiso del editor

RESUMEN Introducción: La fístula faringocutánea post-laringectomía total (LT) continúa siendo una complicación frecuente luego de esta intervención especialmente tras tratamiento radioterápico. Cuando fracasan las medidas conservadoras, es necesario recurrir a cirugía reconstructiva. En nuestro centro se utiliza el colgajo miocutáneo de pectoral mayor (CMPM), que asegura un flujo sanguíneo adecuado para el tratamiento fístulas post-irradiación. Descripción: Presentamos un paciente con una fístula faringocutánea tras LT que requirió una amplia reconstrucción mediante CMPM asimismo discutiremos el importante papel que juega el tratamiento con oxígeno hiperbárico en el manejo de las consecuencias post-radioterapia. Conclusión: En la era de la reconstrucción microvascular, el PMMC puede ser utilizado para la reconstrucción de grandes defectos de cabeza y cuello, consiguiendo una adecuada cobertura tras protocolos de preservación de órgano o pacientes con historia de enfermedad vascular.

PALABRAS CLAVE Pectoral mayor, colgajo pediculado, fístula, laringectomía, reconstrucción, terapia con oxígeno hiperbárico y complicaciones

SUMMARY

Introduction: Pharyngocutaneous fistula after total laryngectomy (TL) remains a hardly frequent complication especially after radiotherapy. When conservative measures fail, reconstructive procedures are necessary. Our institution has adopted the pharyngeal interposition graft (PIG) using a pectoralis major myocutaneous flap (PMMC), which has adequate blood flow, for 

preservation protocols or patiens with a history of vascular disease.

Pectoralis major, pedicled flap, fistula, laryngectomy, reconstruction, hyperbaric oxygen therapy and complications

\section{INTRODUCCIÓN}

En la actualidad es frecuente que se recurra a realizar una laringectomía total (LT) tras fracasos de tratamientos combinados con radioterapia (RT) y quimioterapia (QT). El estado de los tejidos puede condicionar, en algunos pacientes, grandes defectos faringocutáneos. Su reconstrucción supone un desafío para el cirujano, ya que debe asegurar la preservación de la función deglutoria del paciente. Las opciones reconstructivas son diversas, pero complejas, no están exentas de complicaciones y riesgos para el paciente, los cuales se ven incrementados por la desvitalización de tejidos como consecuencia de los tratamientos previos antes mencionados [1].

El colgajo miocutáneo de pectoral mayor (CMPM) ha sido considerado de elección para las reconstrucciones en cabeza y cuello gracias a su proximidad a la región receptora, la fiabilidad de su pedículo y por presentar una corta curva de aprendizaje. Todo ello ha contribuido a su uso generalizado con buenas tasas generales de éxito en servicios de otorrinolaringología (ORL) [2].

Por tanto, hoy en día la reconstrucción faringocutánea mediante CMPM tras LT, después de protocolos de preservación de órgano, supone uno de los principales recursos para la reconstrucción de este tipo de defectos [3].

\section{DESCRIPCIÓN}

Paciente varón de 66 años, fumador y diabético, con antecedente de neoplasia de laringe T3N2M0 tratada con QT y RT concomitante en 2009, que acudió a los 10 años a una revisión rutinaria apreciándose recidiva a nivel glotosupraglótico. En el estudio de extensión, se constató la ausencia de metástasis ganglionares y a distancia. El cuello del paciente presentaba un aspecto leñoso previo a la cirugía. Se realizó una LT, informándose de un carcinoma escamoso moderadamente diferenciado que afectaba al lado izquierdo de glotis y cara laríngea de epiglotis, con bordes de resección libres de neoplasia, sin necesidad de tratamiento oncológico adyuvante. Durante la intervención se constató una evidente afectación de los tejidos con fibrosis extrema e importante desestructuración anatómica.

Al quinto día postquirúrgico, comenzó a observarse una dehiscencia de la sutura, que a las dos semanas progresó a un faringostoma grado IV. Tras 30 sesiones de cámara hiperbárica y curas diarias, presentó mejoría de la vascularización del tejido faríngeo (Figura 1). En este momento, nos planteamos realizar la reconstrucción del defecto faringocutáneo mediante un colgajo miocutáneo de pectoral mayor (CMPM).

Tres meses después de la LT se realizó la reconstrucción, utilizando como variante a la técnica clásicamente descrita, la misma isla cutánea tanto para el cierre faríngeo como para el cierre cutáneo, sin necesidad de utilizar un injerto libre de piel para cubrir el defecto epitelial cervical (Figura 2).

Al quinto día de la intervención presentó una dehiscencia del margen inferior derecho (Figura 3), precisando nuevamente sesiones de cámara hiperbárica. A la décima sesión se constató un cierre completo del defecto (Figura 4). 


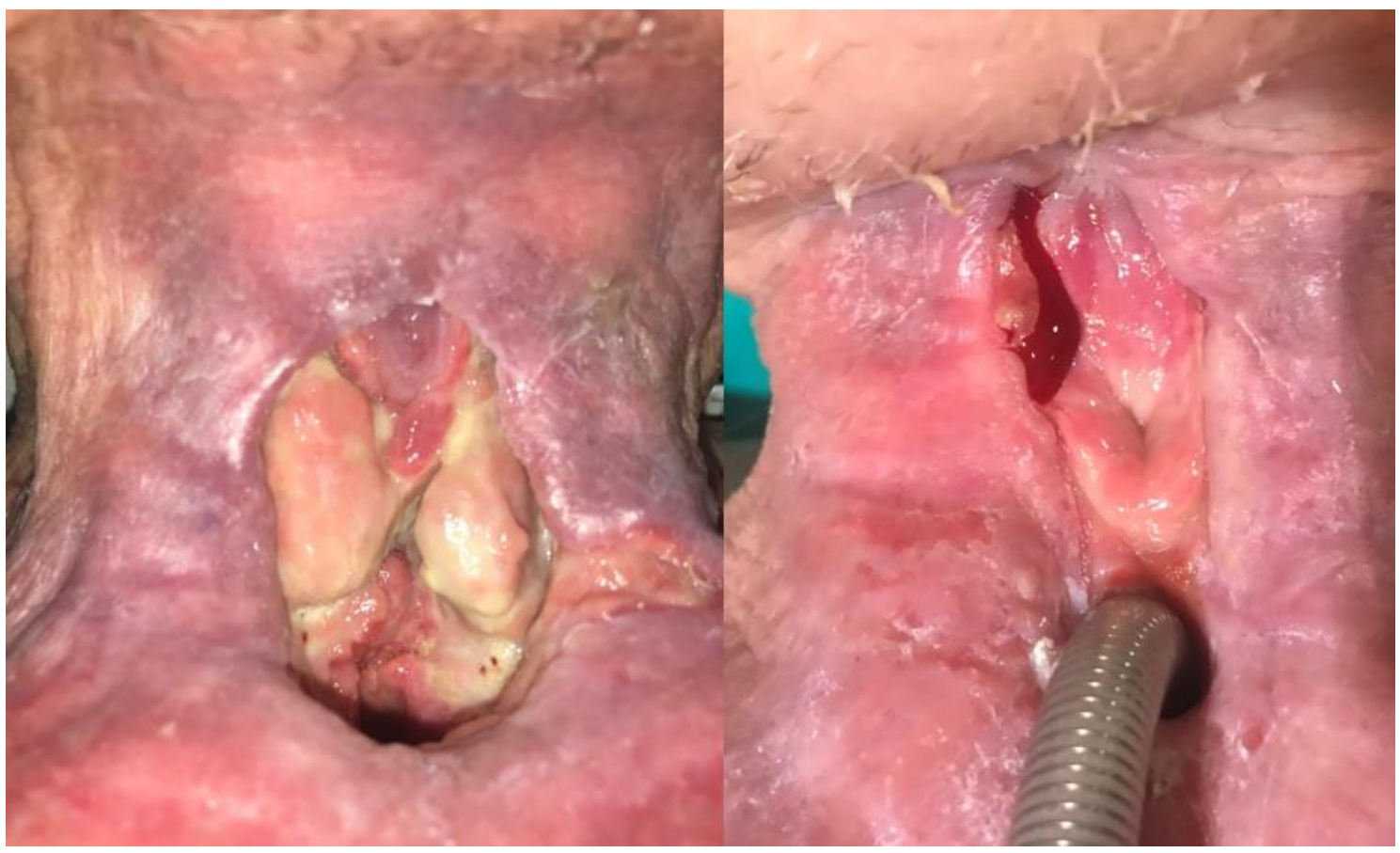

Figura 1. Se aprecia el gran defecto faringocutáneo que presentaba el paciente y su estado previo a la intervención, con mejoría tras las sesiones de oxígeno hiperbárico.

\section{DISCUSIÓN}

La aparición de una fístula faringocutánea es la complicación más común tras la laringectomía total. Esta incrementa la morbilidad, el tiempo de hospitalización y retrasa cualquier tratamiento adyuvante que requiera el paciente. Su incidencia se ha elevado debido al aumento de las cirugías de rescate tras los protocolos de preservación de órgano [4], ya que la QT y RT producen fibrosis, complican la cicatrización y generan trastornos del flujo sanguíneo, por lo que éstas y otras complicaciones (infecciones, dehiscencias, etc) surgen con mayor facilidad [3,5].

El CMPM ha sido clásicamente utilizado en cirugía reconstructiva de cabeza y cuello y de pared torácica anterior desde que Ariyan lo describió por primera vez en 1979 [6]. Gracias al avance de la microcirugía vascular, los colgajos libres son la tendencia en la actualidad, sin embargo, no debemos olvidar que el CMPM posee ventajas como la proximidad al cuello, su versatilidad, la posibilidad de obtener gran cantidad de tejido, su viabilidad, tiempo quirúrgico corto, corta curva de aprendizaje, y además, se presenta como una buena alternativa en los casos en los que los colgajos libres se necrosen $[3,7,8]$. Aún se mantiene la indicación primaria en aquellos casos en los que se necesitan grandes colgajos (por ejemplo, en la reconstrucción tras una glosectomía total), en pacientes en los que haya que proteger los grandes vasos cervicales y, por su puesto, en aquellos centros donde tengan gran experiencia en la utilización de los mismos o servicios de ORL que no cuenten con el apoyo de otras especialidades, como cirugía plástica [8].

Es importante saber que los colgajos libres tienen mayor riesgo de que la anastomosis no sea funcionante en tejidos radiados y en pacientes diabéticos. Por otro lado, este procedimiento incrementa ampliamente el tiempo de cirugía $[5,9]$. En nuestro centro, coincidiendo con otras series como Kekatpure et al [3], Okamoto et al [5] y Righini et al [9], abogamos por la utilización de este tipo de técnica, y la seguimos utilizando ya que es con la que mayor experiencia y menor tasa de complicaciones contamos. 


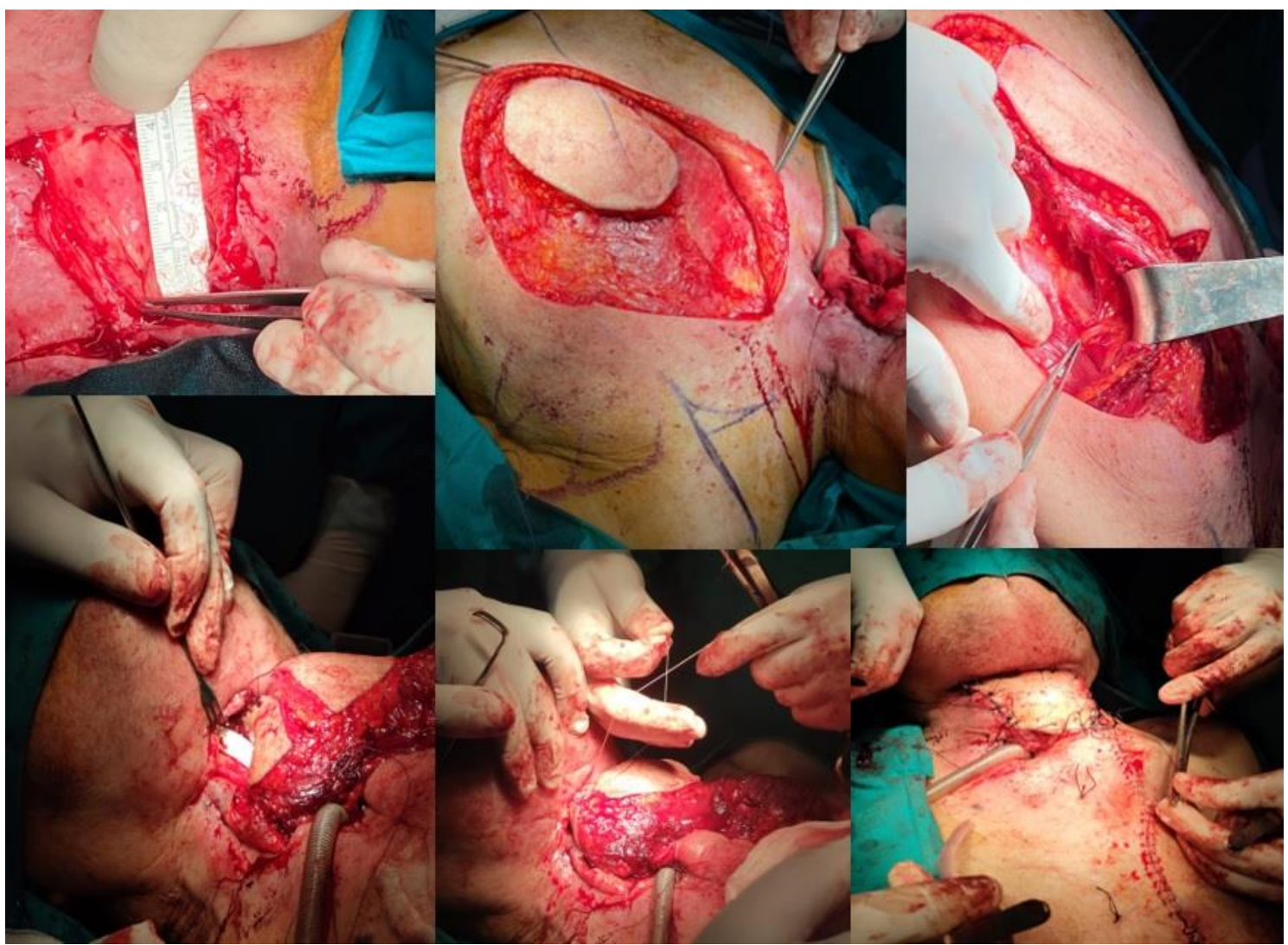

Figura 2. Imágenes de la técnica quirúrgica. Medición del defecto. Creación de la isla cutánea. Exposición del pedículo vascular. Cierre faríngeo utilizando la región inferior de la paleta cutánea. Cierre del defecto cutáneo con el resto del colgajo. Resultado final.

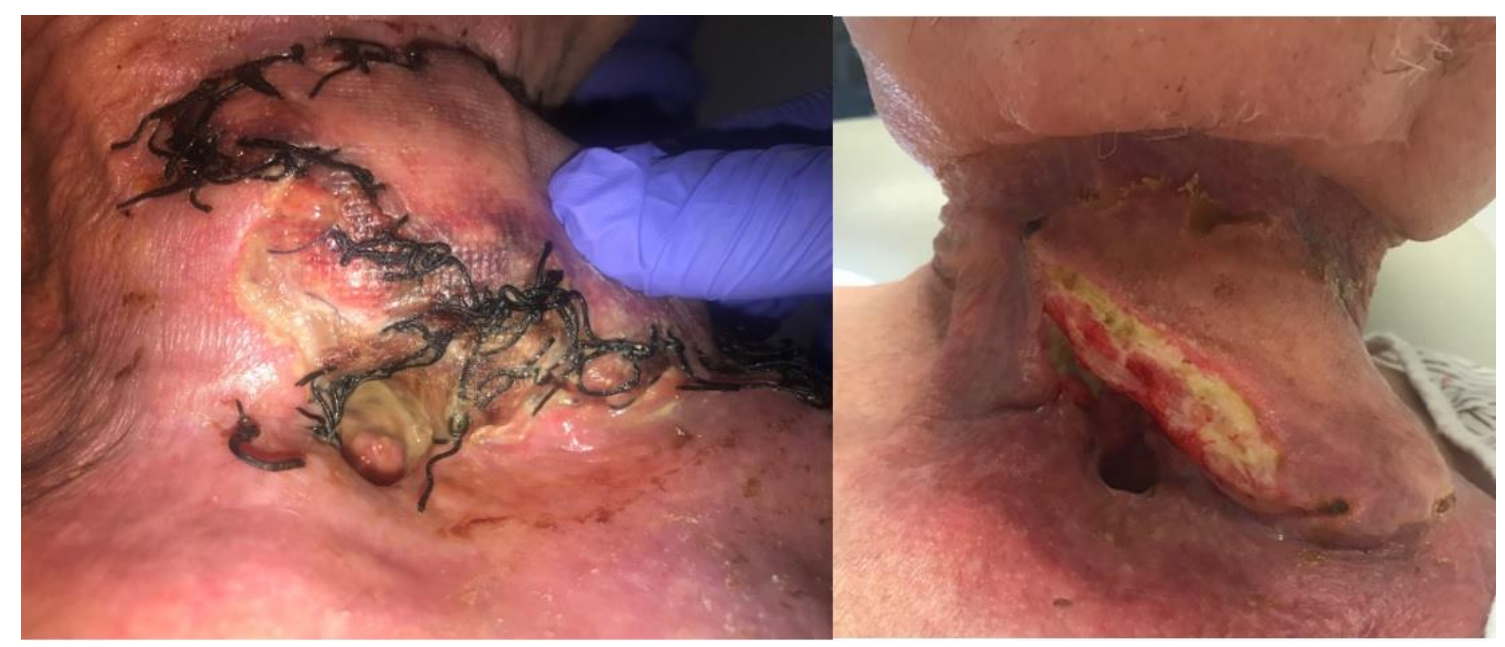

Figura 3. Dehiscencia de la sutura posterior a la intervención.

La isla cutánea debe tener un tamaño suficiente, de forma que incluya ramas perforantes que garanticen su viabilidad, ya que su minimización supone riesgo de

necrosis de la misma. Ésta la diseñamos en posición cráneo-caudal a la areola, disecando hasta el origen del pedículo vascular, que se identifica en su porción más superior (arteria 
toracoacromial), y se envía al sitio receptor a través de un túnel submuscular sobre la clavícula. Esta última maniobra evita lesiones claviculares con la consecuente disfunción del hombro [8].

En nuestro caso, necesitábamos reconstruir un amplio espacio faríngeo y cubrir un extenso defecto cutáneo. Por ello, elegimos una variante de la técnica que consiste en utilizar la misma paleta miocutánea para ambos cierres. En esta alternativa, se reavivan los bordes epiteliales y faríngeos y se divide la paleta en dos, desepitelizando una franja entre ellos, comenzando la sutura por la región más caudal del colgajo para el cierre faríngeoesofágico. La parte superior se pliega sobre sí misma, utilizándose para el cierre epitelial. La sutura se realiza entre la capa submucosa del lecho receptor (esófago o faringe) y la dermis del injerto, con sutura reabsorbible y puntos invertidos [1].

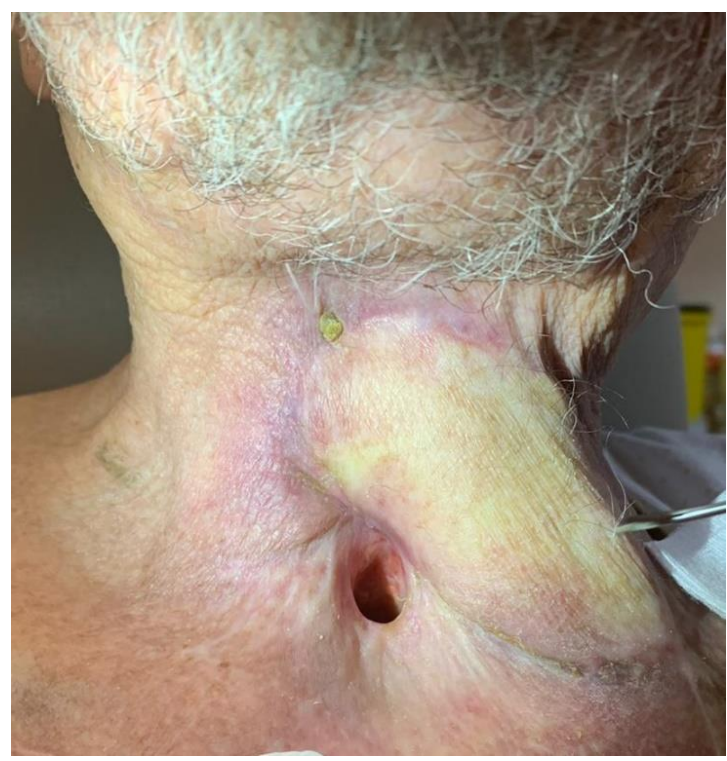

Figura 4. Resultado final tras nuevas sesiones de tratamiento con oxígeno hiperbárico.

La asimetría del complejo areola-pezón es la consecuencia visual más relevante de un CMPM, especialmente importante en pacientes de sexo femenino [1,3]. Además, no está exenta de complicaciones, siendo la dehiscencia de la sutura, con la consecuente fístula salival, la más frecuente [2,3]. No obstante, esta complicación suele poder manejarse de forma conservadora [3].
Actualmente está generalizado el uso de la RT para el tratamiento de tumores malignos de cabeza y cuello. Ésta provoca un entorno hipóxico e hipocelular que acaba dañando el tejido sano circundante, causante de los principales efectos secundarios que influirán en la calidad de vida del paciente. La terapia con oxígeno hiperbárico es utilizada para prevenir y tratar estos efectos, favoreciendo la regeneración tisular [10].

Está claramente demostrado que el oxígeno hiperbárico es de gran utilidad en la supervivencia de colgajos comprometidos ya que ayuda a una mejor oxigenación, mejoría en la función de los fibroblastos, aumenta la síntesis de colágeno, incrementa la angiogénesis y mejora los procesos de isquemia-reperfusión $[11,12]$.

\section{CONCLUSIONES}

El colgajo de pectoral mayor puede ser utilizado de forma segura en cirugías de cabeza y cuello que precisen reconstrucciones amplias, así como aquellos que presentan factores de riesgo elevados para la realización de colgajos libres.

Mediante este caso clínico pretendemos ilustrar cómo un gran defecto faringocutáneo, con tejido desvitalizado amplio, ha podido ser tratado mediante cámara hiperbárica y una variación de la técnica quirúrgica clásica, permitiendo el uso de la misma paleta musculocutánea para evitar un injerto de piel libre.

En nuestro centro no disponemos de servicios como cirugía plástica o cirugía maxilofacial, por lo que recurrimos a esta técnica con asiduidad, contando con una amplia experiencia tanto en su realización como en los cuidados posquirúrgicos de este tipo de pacientes en la planta de hospitalización.

Consideramos decisiva la utilización de cámara hiperbárica para éste y otros pacientes de similares características tratados en nuestro hospital. Pese a una correcta técnica quirúrgica, nos encontramos con un tejido tratado previamente con radioterapia, en un paciente gran fumador y con vasculopatía 
que posee una irrigación tisular seriamente comprometida.

\section{BIBLIOGRAFÍA}

1. Zhang $Y X$, Li Z, Grassetti L, Lazzeri D, Nicoli F, Zenn MR, et al. A new option with the pedicle thoracoacromial artery perforator flap for hypopharyngeal reconstructions. Laryngoscope. 2016: 126(6):1315-20

2. Leite AK, de Matos LL, Belli M, et al. Pectoralis major myocutaneous flap for head and neck reconstruction: risk factors for fistula formation. Acta Otorhinolaryngol Ital. 2014;34:389-93.

3. Kekatpure VD, Trivedi NP, Manjula BV, et al. Pectoralis major flap for head and neck reconstruction in era of free flaps. Int $\mathrm{J}$ Oral Maxillofac Surg 2012;41:453-7.

4. Guimarães AV, Aires FT, Dedivitis RA, et al. Efficacy of pectoralis major muscle flap for pharyngocutaneous fistula prevention in salvage total laryngectomy: A systematic review. Head Neck. 2016;38 Suppl $1:$ E2317-E2321

5. Okamoto I, Tsukahara K, Shimizu A, Sato $\mathrm{H}$. Post-operative complications of salvage total laryngectomy forpost-radiotherapy recurrent laryngeal cancer using pectoralis major myocutaneous flaps. Acta Otolaryngol. 2019; 139:167-71.

6. Ariyan S. The pectoralis major myocutaneous flap. A versatile flap for reconstruction in the head and neck. Plast Reconstr Surg 1979;63:73-81.

7. Teo KG, Rozen WM, Acosta R. The pectoralis major myocutaneous flap. J Reconstr Microsurg. 2013;29:449-456.

8. Liu MY, Liu WW, Yang YH, et al. Pectoralis major myocutaneous flap for head and neck defects in the era of free flaps: harvesting technique and indications. Sci Rep 2017;7:46256.

9. Righini C, Lequeux T, Cuisnier O, Morel N, Reyt E. The pectoralis myofascial flap in pharyngolaryngeal surgery after radiotherapy. Eur Arch Otorhinolaryngol. 2005;262:357-361.
10.Spiegelberg L, Djasim UM, van Neck HW, Wolvius EB, van der Wal KG. Hyperbaric oxygen therapy in the management of radiation-induced injury in the head and neck región: a review of the literatura. J Oral Maxillofac Surg. 2010;68:1732-9.

11.Francis A, Baynosa RC. Hyperbaric Oxygen Therapy for the Compromised Graft or Flap. Adv Wound Care (New Rochelle). 2017;6(1):23-32.

12.Ravi P, Vaishnavi D, Gnanam A, Krishnakumar Raja VB. The role of hyperbaric oxygen therapy in the prevention and management of radiation-induced complications of the head and neck - a systematic review of literature. J Stomatol Oral Maxillofac Surg. (2017) 118:359-62. 